\title{
Role of neutrophil CD11b expression in diagnosis of early- onset neonatal sepsis in full-term infant
}

\section{To the editor,}

Neonatal sepsis is a major cause of death or disability in neonatal age population despite suitable therapy and optimal care. ${ }^{1)}$ The early recognition and diagnosis of neonatal sepsis are difficult because of the nonspecific clinical presentation that mimics other illnesses. For fear of neglecting an actual case of sepsis, antibiotics are administered to all suspected septic neonates. ${ }^{2)}$ This empiric therapy may result in antimicrobial overexposure and resistance and increase healthcare costs.

A definite diagnosis of sepsis is made by the isolation of pathogenic organisms by blood culturing. However, the procedure is time consuming and its sensitivity is suspected to be low. A number of cell surface antigens have been used as diagnostic markers of neonatal sepsis. ${ }^{3)}$ Cluster differentiation (CD) 11b, a member of the $\beta$-integrin family of adhesion proteins, is expressed at very low levels on the surfaces of unstimulated neutrophils. ${ }^{4}$

Our research question was as follows: Can CD11b detect sepsis in full-term infants with suspected sepsis? We hypothesized that the expression of CD11b would be increased in full-term neonates with early-onset sepsis. The study aimed to evaluate the diagnostic value of neutrophil CD11b in the early diagnosis of sepsis in full-term newborn infants (as the majority of studies focused on preterm infants) and determine the correlation between $\mathrm{CD} 11 \mathrm{~b}$ expression and conventional markers as Creactive protein (CRP) and erythrocyte sedimentation rate (ESR) in the diagnosis of neonatal sepsis. The study also examined the diagnostic utility of CD11b combined with CRP and ESR.

This analytical case-control study included 75 full-term infants who were hospitalized in neonatal intensive care unit (NICU) of Al-Zahraa University Hospital. The patients were classified into three groups: Sepsis group $(n=25)$, neonates with proven sepsis based on clinical manifestations of sepsis and positive blood culture; Suspected sepsis group ( $\mathrm{n}=25)$, neonates with suspected sepsis based on strong persistent clinical signs of infection, elevated CRP and ESR, and negative blood culture; and Control group $(n=25)$ neonates with no clinical signs and symptoms suggestive of sepsis, no maternal risk factors, and normal CRP level.

Exclusion criteria included:

- Congenital anomalies

- Inborn error of metabolism

- Birth asphyxia

- Administration of antibiotics prior to inclusion
All groups underwent history taking, clinical examination, and laboratory investigations including complete blood count, CRP, and ESR. CD11b levels were measured using enzyme-linked immunosorbent assay. The statistical analysis was done using IBM SPSS Statistics ver. 25.0 (IBM Co., Armonk, NY, USA).

Our study revealed no significant intergroup differences in gestational age (GA), sex, admission weight, head circumference, body length, or postnatal age among the study groups.

The percentage of neutrophils expressing CD11b was significantly upregulated in the sepsis and suspected sepsis groups versus the control group. Our results were similar with those of previous studies. $\left.{ }^{5,6}\right) \mathrm{CD} 11 \mathrm{~b}$ increases markedly within a few minutes after the cell encounters bacteria or endotoxins. This unique property enables the use of CD11b as an early warning marker for the detection of bacterial infection. ${ }^{7)}$

In this study, CD11b levels were higher in the sepsis group than in the suspected sepsis group; this finding was contradictory to those reported by Adib et al. $\left.{ }^{4}\right)$ This contradiction might be due to differences in the timing of blood collection in relation to infection phase.

The best cutoff point of CD11b was $>0.695 \mathrm{ng} / \mathrm{ml}$; it had a sensitivity, specificity, positive predictive value (PPV), and negative predictive value (NPV) of $100 \%$ in the sepsis group. In the suspected sepsis group, CD11b showed $88 \%$ sensitivity and $80 \%$ specificity with a PPV of $81.5 \%$ and an NPV of $87 \%$ at a cutoff point $>2.0009$. Similar values were detected by one study, ${ }^{6}$ ) while other studies reported different values. $\left.{ }^{4}\right)$ The different findings might be explained in several ways, such as differences in $\mathrm{GA}$ or $\mathrm{CD} 11 \mathrm{~b}$ measurement methods.

The total leukocyte count, absolute neutrophil count, and CRP and ESR levels were less useful as markers for early diag. nosis of sepsis. ${ }^{8)} \mathrm{CRP}$ can be used as a late indicator of neonatal sepsis because it increases slightly in the initial disease phase. Unlike CRP, CD11b does not required newly formed protein synthesis. The combination of CD11b with CRP improved the sensitivity and NPV to $100 \%$, but the specificity and PPV remained nearly the same in the suspected sepsis group. The combined use of CD11b and ESR improved the sensitivity and NPV to $100 \%$ and enhanced the specificity and PPV to $96 \%$ and $96.2 \%$, respectively.

This study demonstrated insignificant differences in CD11b expression between male and female newborn infants in the studied groups. CD11b expression showed a significant increase 
in neonates with positive culture compared to neonates with negative culture results; this finding explained the increase in $\mathrm{CD} 11 \mathrm{~b}$ expression in the sepsis group versus the suspected group. This finding agreed with those of Lai et al. ${ }^{9)}$

The absence of a correlation between CD11b and other hematological parameters indicated that $\mathrm{CD} 11 \mathrm{~b}$ is a sensitive marker whose level increased early in the disease process while the other parameters remained unchanged. ${ }^{9)}$

The clinical practice of treating infants with suspected infection using broad-spectrum antimicrobials increases the risk of invasive fungal infection and promotes the development of resistant bacterial strains. The use of a preventive strategy and application of safe standards can decrease the infection rate within the NICU. ${ }^{10)}$

Although the incidence of sepsis is lower in term infants than in preterm infants, the potential for serious adverse outcomes, including death, is of great consequence; thus, caregivers should have a low threshold for evaluation and treatment for possible sepsis in any infant regardless of GA. ${ }^{8}$

The limitation of this study was its lack of including fungal pathogens and classifying bacterial infections into gram-positive or -negative organisms. However, we used other conventional tools such as clinical sepsis score, hematological picture, CRP, and ESR to confirm the diagnosis.

Our findings suggest that CD11b is a sensitive marker for sepsis and suspected sepsis in full-term neonates and that it may be added to sepsis markers. This information would allow the neonatologist to confidently discontinue antibiotic use as long as the neonate is clinically stable.

\section{Key message}

Question: Can CD11b detect sepsis in full-term infants with suspected sepsis?

Finding: The percentage of neutrophils expressing CD11b was significantly upregulated in the sepsis and suspected sepsis groups versus the control group.

Meaning: CD11b is a sensitive marker for sepsis and suspected sepsis in full-term neonates and it may be added to sepsis markers. This information would allow the neonatologist to confidently discontinue antibiotic use as long as the neonate is clinically stable.

The study protocol (No. 382) was approved by the committee council of Faculty of Medicine for Girls, AL-Azhar University (ethical committee No. 202001013).

Informed consent was obtained from the parents after the aim of the study was explained.

\section{Conflicts of interest}

No potential conflicts of interest relevant to this article are reported.

Safaa ELMeneza, $\mathrm{MD}^{1}$, Walaa Mohamed, $\mathrm{MS}^{1}$, Iman Elbagoury, $\mathrm{MD}^{2}$, Karima Bahagat, $\mathrm{MD}^{1}$
${ }^{1}$ Department of Pediatrics, Faculty of Medicine for Girls, ALAzhar University, Cairo, Egypt

${ }^{2}$ Department of Clinical pathology, Faculty of Medicine for Girls, AL-Azhar University, Cairo, Egypt

Corresponding author: Safaa ELMeneza, MD

AL-Azhar University, Faculty of Medicine for Girls, AL-Azhar University Campus Elmokaiam Eldaym Street, Six district, Nasr city 1141, Cairo, Egypt

凶E-mail:safaa5@hotmail.com, safaaelmeneza@azhar.edu.eg https://orcid.org/0000-0002-4853-4648

See the commentary "Neutrophil CD11b as a promising marker for early detection of neonatal sepsis" via https://doi. org/10.3345/cep.2020.00626.

\section{References}

1. Brocklehurst P, Farrell B, King A, Juszczak E, Darlow B, Haque K, et al. Treatment of neonatal sepsis with intravenous immune globulin. N Eng J Med 2011;365:1201-11.

2. Soni S, Wadhwa N, Kumar R, Faridi M, Sharma S, Chopra A, et al. Evaluation of CD64 expression on neutrophils as an early indicator of neonatal sepsis. Pediatr Infect Dis J 2013;32:e33-7.

3. ELMeneza S, Abu-Shady M, Aref M, Abd-ELBaseer A. Neutrophil CD 64 as marker to differentiate early sepsis from noninfectious respiratory disorders in newborn infants. Acta Sci Paediatr 2018;1:10-15.

4. Adib M, Ostadi V, Navaei F, Saheb Fosoul F, Oreizi F, Shokouhi R, et al. Evaluation of $\mathrm{CD} 11 \mathrm{~b}$ expression on peripheral blood neutrophils for early detection of neonatal sepsis. Iran J Allergy Asthma Immunol 2007; 6:93-6.

5. Du J, Li L, Dou Y, Li P, Chen R, Liu H. Diagnostic utility of neutrophil CD64 as a marker for early-onset sepsis in preterm neonates. PLoS One 2014;9:e102647.

6. Nupponen I, Andersson S, Järvenpää AL,Kautiainen H, Repo H. Neutrophil CD11b expression and circulating interleukin-8 as diagnostic markers for early-onset neonatal sepsis. Pediatrics 2001;108:e12.

7. Ng PC, Lam HS. Biomarkers for late-onset neonatal sepsis: cytokines and beyond. Clin Perinatol 2010;37:599-610.

8. EL Meneza SA, Esmail HO, Elbagoury EM, Abd Allah NA. Soluble triggering receptors expressed on myeloid cell- 1 and proadrenomedullin for diagnosis and prognosis of early onset neonatal sepsis. EC Paediatr 2018;7:619-28.

9. Lai YS, Wang CC, Hua YM, Hung CH. Expression of neutrophil CD11b in preterm neonate and neonatal sepsis: a preliminary report. J Med Sci 2005;25:181-4.

10. Egyptian Neonatal Safety Standards [Internet]. Egyptian Neonatal Safety Training Network; 2014 [cited 2019 Feb 2]. Available from: https:/www. researchgate.net/publication/322831357_Egyptian_Neonatal_Safety_ Standards.

How to cite this article: ELMeneza S, Mohamed W, Elbagoury I, Bahagat K. Role of neutrophil CD11b expression in diagnosis of early-onset neonatal sepsis in full-term infant. Clin Exp Pediatr 2021;64:44-5. https://doi.org/10.3345/cep. 2019.01319 